\title{
Diagnosis of Plasmodium falciparum Malaria in Children using the Immuno-Chromatographic Diagnostic Technique
}

\author{
AE Ben-Edet*, FEA Lesi**, AG Mafe ${ }^{+}$, AO Grange***
}

\begin{abstract}
Summary
Ben-Edet AE, Lesi FEA; Mafe AG, Grange AO. Diagnosis of Plasmodium falciparum Malaria in Children using the Immuno-Chromatographic Diagnostic Technique. Nigerian Journal of Paediatrics 2004; 31:00.

Background: Children under five years of age constitute the "at risk" population for fatal complications of P. falciparum malaria infection. Early diagnosis and prompt treatment are key features in the current Roll Back Malaria Programme.

Objective: To assess the diagnostic performance of the Immunochromatographic test (ICT) in the rapid diagnosis of falciparum malaria infection in children aged less than five years with a presumptive clinical diagnosis of malaria.

Method: This prospective study was conducted at the Children Emergency Room of the Lagos University Teaching Hospital between January and October 2001. Febrile children aged less than five years and were suspected to have malaria, were enrolled. Blood samples from all patients were tested using the ICT-Malaria P $\mathrm{f}$ test kit as well as the conventional blood film microscopy after Giemsa staining.

Results: The results showed that the diagnosis of falciparum malaria was achieved within eight minutes using the ICT-Malaria P f o test kit. The sensitivity of the test kit was 69.6 percent while the specificity was 98.4 percent. However, among patients with severe malaria, the ICT had a sensitivity of 90.3 percent: The test kit was unable to detect antigenaernia at parasite density below $400 / \mathrm{ml}$. An increase in body temperature was associated with higher rate of ICT test positivity.

Conclusion: The low sensitivity of the ICT technique obtained in this study makes it a poor screening tool for the routine diagnosis of falciparum malaria in children aged 0-5yrs. It may be useful in the rapid diagnosis of suspected severe malaria in febrile children. The high cost of the test kit is likely to prove a major limitation to its widespread use.
\end{abstract}

Key Words: Malaria diagnosis, Children, ICT test

\begin{tabular}{l}
\hline University of Uyo Teaching Hospital, Uyo \\
Department of Paediatrics \\
*Consultant \\
\hline Lagos University Teaching Hospital, Lagos \\
\hline Department of Paediatrics \\
** Senior Lecturer/Consultant \\
*** Professor \\
\hline Nigerian Institute of Medical Research, Yaba, \\
Lagos \\
${ }^{+}$Chief Medical Laboratory Scientist
\end{tabular}

Correspondence: Dr. FEA Lesi.

Email: afolabilesi@hotmail.com

\section{Introduction}

MALARIA remains a major public health and socioeconomic problem in the world." Globally, between $300-$ 500 million people contract the disease and 1.5-2.7 million people die from it annually. Over 90 percent of these people reside in tropical Africa. ${ }^{1}$ Malaria is one of the major childhood killers in Africa. ${ }^{2}$ An estimated one million children die from the effect of malaria in Africa annually. ${ }^{1}$ In Nigeria, malaria has consistently been among the top three reported causes of deaths among children under five years of age. ${ }^{3}$ In order to prevent diagnostic delays and fatal outcome of malaria in endemic zones, the World Health Organization recommended that every febrile child must be treated for malaria until proven otherwise. ${ }^{4}$ The 
commencement of anti-malarial drugs without a laboratory confirmation is advocated for endemic zones, in order to prevent the fatal consequences of falciparum malaria that may follow a delay. ${ }^{4}$ This recommendation is in recognition of the lack of supportive laboratory facilities, poor electricity and water supply, as well as the paucity of trained technicians to conduct laboratory tests on a regular basis in most endemic zones. ${ }^{5}$

The gold standard for malaria diagnosis is the conventional blood film technique, which is relatively cheap, highly sensitive and specific. However, it is labour intensive and time consuming and not universally available. ${ }^{6-8}$ These obstacles led to an increased dependence on clinical diagnosis and the search for other simpler, cost effective and rapid diagnostic techniques. The newly developed antigen capture techniques, which are now available as test kits (ICT-Malaria Pf ${ }^{\circ}$, Para Sight$F$ Malaria ${ }^{\circ}$ and Optimal I and II test kits), appear promising. These diagnostic options are said to be rapid, with results obtainable within 10 minutes, and simple to conduct by unskilled persons including the mothers, with little or no laboratory experience. They are highly sensitive and specific for falciparum malaria, the lethal specie infecting our children. ${ }^{6,9}$ This would hasten early diagnosis and prompt treatment of children with potentially fatal falciparum malaria. ${ }^{6,10}$

The Roll Back Malaria Programme has suggested the inclusion of these test kits in the package for home treatment of malaria. ${ }^{1}$ The programme recognizes the fundamental role of early, rapid and precise diagnosis of malaria as well as appropriate treatment, in the current global effort to halve malaria deaths. ${ }^{1}$ As such, several rapid diagnostic tests kits have been developed. The $I C T$ Malaria Pf test (ICT) kit, which was used in this study, specifically detects the Histidine Rich Protein II (HRPII) antigen produced only by P. falciparum; this is the newer of the HRP-II test kits. Studies carried out in various malaria-endemic countries indicate that the kits are easy to use, require no special skills, rapid with completion time of about 10 minutes and demonstrate a high diagnostic performance., ${ }^{4,10,12-15}$ The kits were particularly useful in severe falciparum malaria where parasites may be sequestered in the deep blood vessels and may not be found in the peripheral blood by microscopic methods, but the antigens are easily detected by the new HRP-II test system. ${ }^{6,7,9,12}$ Very few studies on ICT-Malaria $\mathrm{P \rho}$ have been reported from Nigeria, probably because the kits are not yet universally available in the country. Such few studies have shown that the ICT-Malaria $P \dot{\rho}$ has high sensitivity (89-96 percent ) and specificity (98-100 percent). ${ }^{6,16}$

We set out to evaluate the diagnostic performance (the sensitivity, specificity, positive and negative predictive values) of the Immuno-chromatographic Diagnostic
Technique (ICT-Malaria Pf $\dot{\partial}$ test) among children under five years of age at the Olikoye Ransome-Kuti Children Emergency Room (ORK-CHER) of the Lagos University Teaching Hospital (LUTH). We also wanted to determine if factors such as previous antimalarial use affected the diagnostic performance of the ICT-Malaria $P \dot{f}$ technique.

\section{Materials and Methods}

This prospective study was conducted at the Lagos University Teaching Hospital at Idi-Araba, between January and October 2001. Ethical approval was obtained from the LUTH Ethical Committee prior to the commencement of the study. Included in the study were children under five years, consecutively presenting at the Olikoye Ransome-Kuti Children Emergency Room (ORK-CHER) with fever (axillary temperature of ${ }^{3} 37.5^{\circ} \mathrm{C}$ ) or a recent ( $<48$ hours) history of fever, or a clinical diagnosis of malaria. Excluded were children with chronic illnesses. The minimum sample size (n) of 323 was calculated for this cross-sectional study using the best estimate population prevalence rate for malaria at thirty percent. ${ }^{16}$ Informed consent was obtained from the parents/guardians of eligible children. Enrolled children had a detailed history taken and a physical examination performed. Information obtained included the age, sex and history of previous anti-malarial use. The temperatures were taken using a Celsius mercury thermometer.

\section{Laboratory Methods}

\section{ICT-Malaria $P F^{o}$ Test}

The ICT-Malaria Pf Test was performed using the ICT-Malaria Pf Test Kit, and interpreted as described by the manufacturers. The kit contains 25 test cards, 25 EDTA coated capillary tubes and one bottle of Reagent A. It has a shelf life of three to five years at the storage temperature of -2 to $+25^{\circ} \mathrm{C}$. As an in-vitro immunodiagnostic test, it detects circulating $\mathrm{P}$. falciparum Histidine-Rich Protein II antigen in whole blood, using two antibodies. One antibody is attached to a visible colloidal gold and impregnated unto a sample pad, while the second antibody is immobilized as a line across the test strip. Approximately $10 \mathrm{ml}$ of whole blood is added to the sample pad where lysis occurs and PF HRP-II antigen if present, binds to the colloidal goldlabelled antibody. When Reagent $A$ is added to the sample pad, blood and labelled antibody migrate up the test strip crossing the second antibody line. In a positive sample, the antibody on the membrane captures $\mathrm{P}$. falciparum HRP-II antigen complexes with the goldlabelled/antibody and a pink line forms. In a negative 
sample, no pink line forms. Antigenaemia (the finding of malaria parasite antigens in blood) was defined by the presence of a pink line on the test pad.

\section{The Conventional Blood Film Technique}

The conventional blood film microscopy (the gold standard for malaria diagnosis) was carried out according to standard procedures using a three percent Giemsa stain solution prepared from a stock of Giemsa stain. Thick and thin blood films were prepared on the same slide for each patient and labelled. The thin film was air dried and fixed with methanol prior to staining. Prepared slides were stained for 30-45 minutes. The films were examined for the presence of malaria parasites, species of the parasite, stages of parasite development, and the parasite density. The total parasite counts were obtained by counting the number of malaria parasites around 200 white blood cells and then multiplying by 40 to give the number of parasites $/ \mathrm{ml}$ (after assuming that there are about $8000 \mathrm{WBC} / \mathrm{ml}$ blood). ${ }^{18}$ The thick smear was considered negative if no parasites were seen after 10 minutes of searching. ${ }^{18}$ The specie of the malaria parasite and its stage of development were noted on the thin film. ${ }^{17,18}$ Parasitaemia was defined as the presence of asexual malaria parasites in the peripheral blood film after Giemsa stain.

Children with acute uncomplicated malaria were treated with chloroquine $(25 \mathrm{mg} / \mathrm{kg} / 3$-day course) as first line and those with acute severe malaria received intravenous quinine $(10 \mathrm{mg} / \mathrm{kg} / \mathrm{dose}$ in 10 percent dextrose water infusion every eight hours until the patient regained consciousness).

\section{Results}

Three hundred and twenty three consecutive children under five years of age were admitted with fever or a history of fever. Table I shows that 130 (40.2 percent) had a clinical (presumptive) diagnosis of acute malaria. Severe malaria was diagnosed in 46 (14.2 percent) children while eight ( 2.5 percent) had a diagnosis of drug resistant malaria. Also diagnosed were 51 (15.8 percent) with acute respiratory infection, 29 ( 9.0 percent) with septicemia, 27 ( 8.4 percent) with gastroenteritis and 32 (9.9 percent) children with a variety of other diagnoses such as meningitis, cellulitis, tonsillitis and tetanus. The outcome in the patients studied is also summarized in Table I. Out of the 323 children studied, 321 (99.4 percent) were discharged either from outpatient or inpatient management after successful treatment. However, two ( 0.6 percent) of the children, one with severe malaria and the other with suspected meningitis, died after admission and commencement of therapy.

Table II shows that there were 84 (26.0 percent) positive Giemsa stained slides in contrast to the 42 (13.0 percent) positive ICT test results, a difference that was. significant $(p=0.00003)$. The table also shows that the Giemsa stain microscopy was more accurate in the confirmation of a clinical diagnosis of acute malaria in 25 (19.2 percent) children out of 130 than the ICT test kit which detected $P$. falciparum antigens in only 11 ( 8.5 percent) of these children; this difference in detection rates was also significant $(p=0.01)$ : Out of the 46 children with suspected severe malaria, 31 (67.4 percent) were parasitaemic while 30 ( 65.2 percent) had positive ICT test results. The clinical diagnosis of drug resistant

Table I

Presumptive Clinical Diagnosis and Outcome

\begin{tabular}{|c|c|c|}
\hline Presumptive Diagnosis & No of Cases & $\%$ of Total \\
\hline Malaria $(n=184)$ & 130 & 40.2 \\
\hline Severe malaria & 46 & 14.2 \\
\hline Drug resistant malaria & 8 & 2.5 \\
\hline Acute respiratory infection & 51 & 15.8 \\
\hline Septicaemia & 29 & 9.0 \\
\hline Gastroenteritis & 27 & 8.4 \\
\hline Meningitis & 15 & 4.6 \\
\hline Miscellaneous & 17 . & 5.3 \\
\hline Total & 323 & 100.0 \\
\hline
\end{tabular}

Outcome

Discharged

Died

3212

$99.4 \quad 0.6$ 
Table II

A Cross-Tabulation of the Clinical Diagnosis against ICT-Malaria P户े Test and Giemsa Stain Microscopy Results

\begin{tabular}{lccccl}
\hline Clinical Diagnosis & \multicolumn{2}{c}{ Giemsa Stain Microscopy } & \multicolumn{2}{c}{ ICT Test } & \\
\cline { 2 - 5 } & Positive (\%) & Negative (\%) & Positive (\%) & Negative (\%) & P-Value \\
\hline Acute malaria( $\mathrm{n}=130)$ & $25(19.2)$ & $105(80.8)$ & $11(8.5)$ & $119(91.5)$ & 0.01 \\
Severe malaria $(\mathrm{n}=46)$ & $31(67.4)$ & $15(32.6)$ & $30(65.2)$ & $16(34.8)$ & 0.8 \\
Drug resistant malaria $(\mathrm{n}=8)$ & 0 & $8(100.0)$ & 0 & $8(100.0)$ & - \\
ARI $(\mathrm{n}=51)$ & $6(11.8)$ & $45(88.2)$ & $1(2.0)$ & $50(98.0)$ & 0.1 \\
Septicaemia $(\mathrm{n}=29)$ & $9(31.0)$ & $20(69.0)$ & 0 & $29(100.0)$ & 0.003 \\
Gastroenteritis $(\mathrm{n}=27)$ & $6(22.2)$ & $21(77.8)$ & 0 & $27(100.0)$ & 0.03 \\
Meningitis $(\mathrm{n}=15)$ & $2(13.3)$ & $13(86.7)$ & 0 & $15(100.0)$ & 0.5 \\
Miscellaneous $(\mathrm{n}=17)$ & $5(29.4)$ & $12(70.6)$ & 0 & $17(100.0)$ & 0.052 \\
\hline Total $(\mathrm{n}=323)$ & $84(26.0)$ & $239(74.0)$ & $42(13.0)$ & $281(87.0)$ & 0.00003 \\
\hline
\end{tabular}

$\mathrm{ARI}=$ Acute respiratory infection

Table III

A Cross-Tabulation of Results of Microscopy with Giemsa Stain and ICT Tests

\begin{tabular}{llll}
\hline ICT Test Results & \multicolumn{2}{c}{ Results of Microscopy with Giemsa Stain } & Total \\
\cline { 2 - 4 } & Positive & Negative & \\
\hline Positive & TP & FP & \\
& $39(21.2)$ & $2(1.1)$ & $41(22.3)$ \\
Negative & FN & TN & \\
& $17(9.2)$ & $126(68.5)$ & $143(77.7)$ \\
\hline Total & $56(30.4)$ & $128(69.6)$ & $184(100.0)$ \\
\hline
\end{tabular}

TP $=$ True Positive (i.e. positive by both microscopy and ICT)

$\mathrm{FP}=$ False Positive (i.e. positive by ICT but not by microscopy)

$T N=$ True Negative (i.e. negative by both microscopy and ICT)

$\mathrm{FN}=$ False Negative (i.e. negative by ICT but positive by microscopy)

malaria was not confirmed by either laboratory methods as shown in Table II. In children in whom clinical diagnoses other than malaria were made, the microscopy of Giemsa stained blood films detected varying degrees of parasitaemia but none except one child with acute respiratory infection (ARI) had antigenaemia detected by ICT.

Table III shows that out of the 184 children who had a presumptive diagnosis of malaria, 56 (30.4 percent) had positive Giemsa stained slides while the ICT test kit identified 41 (22.3 percent) children with antigenaemia. The table also shows that 39 (21.2 percent) patients were positive (True Positive) and 126 (68.5 percent) negative (True Negative) by both techniques. However, there were two (1.1 percent) patients with negative Giemsa stained slides but positive ICT test results (False Positive), while 17 ( 9.2 percent) had positive Giemsa stained slides but negative ICT test 
Table IV

The Diagnostic Performance of the ICT-Malaria Pf Test Kits

\begin{tabular}{lrc}
\hline Diagnostic Performance Indices & Results & $(\%)$ \\
\hline Sensitivity & $39 / 56$ & $(69.6)$ \\
Specificity & $126 / 128$ & $(98.4)$ \\
Positive Predictive Value & $39 / 41$ & $(95.1)$ \\
Negative Predictive Value & $126 / 143$ & $(88.1)$ \\
False Positive Rate & $2 / 128$ & $(1.6)$ \\
False Negative Rate & $17 / 56$ & $(30.4)$ \\
Accuracy Rate & $41 / 56$ & $(73.2)$ \\
\hline
\end{tabular}

Table V

Effects of Some Factors on the Diagnostic Performance of the ICT-Malaria P户 Test Kits

\begin{tabular}{|c|c|c|c|c|c|c|}
\hline \multirow{2}{*}{ Factor } & \multicolumn{3}{|c|}{$I C T$} & \multicolumn{3}{|c|}{ Giemsa } \\
\hline & $\begin{array}{l}\text { Positive } \\
(N=41) \\
n(\%)\end{array}$ & $\begin{array}{l}\text { Negative } \\
(N=143) \\
n(\%)\end{array}$ & pualue & $\begin{array}{l}\text { Positive } \\
N=56) \\
n(\%)\end{array}$ & $\begin{array}{l}\text { Negative } \\
N=128) \\
n(\%)\end{array}$ & p value \\
\hline Male sex $(n=109)$ & $23(56.1)$ & $86(60.1)$ & 0.7 & $30(53.6)$ & $79(61.7)$ & 0.4 \\
\hline Temperature $37.5(n=140)$ & $37(90.2)$ & $103(72.0)$ & 0.03 & $46(82.1)$ & $94(73.4)$ & 0.3 \\
\hline Prior antimalarial use $(n=84)$ & $18(43.9)$ & $66(46.2)$ & 0.9 & $26(46.4)$ & $58(45.3)$ & 0.9 \\
\hline Age $<12$ months $(n=47)$ & $6(14.6)$ & $42(29.4)$ & 0.09 & $8(14.3)$ & $39(30.5)$ & 0.03 \\
\hline
\end{tabular}

results (False Negative). The diagnostic performance of the test kit-ICT was then computed from the values in Table III. It is noted that 10 out of the 184 children had P. malariae detected. However none were positive by IC'T test (not shown in the Table). The results summarized in Table IV show that the ICT - Malaria P $f \dot{O}$ technique, had a sensitivity of 69.6 percent and a specificity of 98.4 percent; the positive predictive value was 95.1 percent while the negative predictive value was 88.1 percent. The false positive and false negative rates were 1.6 percent and 30.4 percent, respectively. The accuracy rate of the ICT test kit against the conventional microscopy method was 73.2 percent.

Some factors that might possibly affect the diagnostic performance of the test kit such as the age of the child, sex, body temperature at the time of the test and antimalarial drug use prior to the test were reviewed and shown on Table V. It shows that temperatures greater than $37.5^{\circ} \mathrm{C}$ at the time of the ICT test was capable of increasing the number of positive results $(p=0.03)$ as compared with Giemsa stain technique which did not seem to be influenced by temperature $(p=0.3)$.
Furthermore, in children aged less than 12 months, there were significantly more positive Giemsa slides $(p=0.03$ ) than positive ICT results $(p=0.3)$. However, neither the gender of the patients nor the use of antimalarial drugs prior to the test had any significant effect on the results.

A review of the parasite density detected by ICT technique (Table VI) shows that in two of the patients, there were no parasites detected but the ICT test was positive. A further review of these two cases showed that a clinical diagnosis of acute severe malaria wàs made and both had received antimalarial drugs (quinine and chloroquine) prior to the tests. Apart from these two cases, the test kit was only 14.3 percent accurate at parasite counts below $400 / \mathrm{ml}$. The accuracy rate increased to 72.7 percent at counts greater than $401 /$ $\mathrm{ml}$. At parasite counts above 3,201-parasite $/ \mathrm{ml}$, the test kit was able to detect P. falciparum HRP-II antigen in all the samples which were positive by Giemsa stain microscopy. The performance time for each Giemsa stain microscopy was about 60 minutes compared to a performance time of eight minutes for the ICT test. The average cost of the Giemsa stain microscopy was 
Table VI

A Cross-Tabulation of Malaria Parasite Counts with the ICT Test Results

\begin{tabular}{llll}
\hline $\begin{array}{l}\text { Malaria } \\
\text { Parasite Counts }\end{array}$ & \multicolumn{2}{c}{ ICT Test Results } & Total (\%) \\
\cline { 2 - 3 } & Positive (\%) & Negative (\%) & \\
\hline None & $2(1.6)$ & $126(98.4)$ & $128(100.0)$ \\
$101-200$ & 0 & $3(100.0)$ & $3(100.0)$ \\
$201-400$ & $1(14.3)$ & $6(85.7)$ & $7(100.0)$ \\
$401-800$ & $8(72.7)$ & $3(27.3)$ & $11(100.0)$ \\
$801-1,600$ & $7(70.0)$ & $3(30.0)$ & $10(100.0)$ \\
$1,601-3,200$ & $3(60.0)$ & $2(40.0)$ & $5(100.0)$ \\
$3,201-6,400$ & $1(100.0)$ & 0 & $1(100.0)$ \\
$6,401-12,800$ & $3(100.0)$ & 0 & $3(100.0)$ \\
$12,801-25,600$ & $14(100.0)$ & 0 & $14(100.0)$ \\
$>25,600$ & $2(100.0)$ & 0 & $2(100.0)$ \\
\hline Total & $41(22.3)$ & $147(77.7)$ & $184(100.0)$ \\
\hline
\end{tabular}

$c^{2}=132.3, p=0.00001$

N150 (US\$1.5) per test compared with N280 (US\$2.8) per ICT test.

\section{Discussion}

This study shows that out of the 130 children with a presumptive clinical diagnosis of acute malaria, 91.5 percent were ICT negative while 80.8 percent were Giemsa stain microscopy negative. This suggests that while malaria is a common presumptive diagnosis in our children with fever, it is most often inaccurate in at least, 80 percent of children under five years. This observation has been made by others who have reported inaccuracy rates ranging between 46.1 percent and 74 percent. ${ }^{20-22}$ This implies that the presumptive diagnosis of malaria may lead to inappropriate treatment, inadequate investigation into other possible causes of illness and delay in administration of appropriate therapy as has been noted by other workers. ${ }^{20,22,23}$. The presumptive clinical diagnosis of severe malaria in 46 children was more accurate, with lower inaccuracy rate of 34.8 percent and 32.6 percent by ICT and Giemsa stain microscopy, respectively. It is noteworthy that the clinical diagnosis of drug resistant malaria in eight children was 100 percent inaccurate, as no laboratory basis was found for this diagnosis. This shows the inherent danger of treatment based on clinical diagnosis alone, as these eight children could have received a course of another antimalarial drug, without full investigations into the possible causes of their illnesses. This reinforces the accepted practice that the diagnosis of drug resistant malaria should never be made on clinical grounds alone without relevant laboratory support. Also, varying degrees of parasitaemia were detected in some of the children in the study in whom other clinical diagnoses such as ARI, gastroenteritis, meningitis etc, were made. Only one child with ARI was found to be antigenaemic. Asymptomatic parasitaemia is a common finding in endemic zones, ${ }^{24}$ the finding of parasitaemia without antigenaemia in these children is therefore not surprising. It confirms the observation that several other illnesses may co-exist with, or mimic malaria. ${ }^{24,25}$

P. falciparum antigenaemia by the ICT technique was found in 22.3 percent of the 184 patients studied. Asianya et al, in a similar study in both children and adults, obtained positive ICT results in 16.2 percent of 105 children. ${ }^{16}$ However, the ages of the children were not specified, making comparisons difficult. Other reported studies with ICT were carried out in adults. ${ }^{6,13-15}$ When the ICT results were compared with Giemsa stained blood film microscopy which is the diagnostic gold standard, there were more children with parasitaemia [56 (30.4 percent)] than antigenaemia [41 (22.3 percent)], giving an accuracy rate of 73.2 percent for the ICT test. This degree of accuracy does not compare with a figure of 95 percent reported from Thailand. ${ }^{15}$ Not all children who tested positive with Giemsa stain microscopy were positive by ICT; these were false negative cases by ICT -17 (9.2 percent) children. The false negative rate was 
30.4 percent. The large number of false negatives may lead to the failure to diagnose falciparum malaria in many children in this age group, with fatal consequences. These false negative cases were found to have parasite counts below $400 / \mathrm{ml}$. In this study, the ICT kit did not detect antigenaemia at parasite counts $<201 / \mathrm{ml}$ and detected only one ( 14.3 percent) sample, out of the six with parasite count of $201-400$ parasite $/ \mathrm{ml}$. This may have contributed to the high false negative rate and low sensitivity obtained. Conversely, there were two (1.1 percent) ICT positive samples that were negative by the Giemsa stain microscopy technique. These were false positive results in two children with the clinical diagnosis of severe malaria. Both febrile children had received some doses of quinine and chloroquine prior to the tests. This finding of antigenaemia in the absence of parasitaemia, suggests that the parasites may have sequestered into the deep vessels in the two children with severe malaria. ${ }^{6,9,26}$ The persistence of antigenaemia after a cure is well documented as a limitation to the rapid diagnostic antigen test. However, in these two cases, it is possible that the antimalarial drugs received prior to the test, had grossly reduced the parasite density to levels that were not detected by microscopy. ${ }^{6}, 10,12$

The sensitivity of the ICT test kit in this study involving under-five children was 69.6 percent. This is low, when compared with other studies which reported sensitivities of 86-96 percent. .,10,13-16 $_{\text {However, it is }}$ noteworthy that apart from the study by Asianya et al, that included children of unspecified ages, ${ }^{16}$ other studies involved adults only. ${ }^{6,10,13 \cdot 15}$ The specificity, positive predictive value, and false positive rate of 98.4 percent, 95.1 percent and 1.7 percent respectively, in this study, are similar to the reported specificities of 98-100 percent and positive predictive values of $96-100$ percent obtained in other series. ${ }^{6,10,13-16}$ The large number of false negative results obtained in our study might have contributed to the low sensitivity, as the ICT test did not detect low levels of parasitaemia. This has also been documented by others who noted sensitivities as low as 23 percent in patients when parasite counts were less than $500 / \mathrm{ml}$; most of these patients were asymptomatic. ${ }^{27}$ In spite of the above, the ICT technique does have some advantages over the Giemsa microscopy technique. The ICT performance time was eight minutes compared to the 60 minutes for Giemsa stain microscopy. Other studies on ICT have reported a mean performance time of ten minutes as opposed to the 60 minutes for microscopy with Giemsa stain., ${ }^{6,10,13-16}$ As documented in other studies, the procedure for ICT in this study was simple to perform, the result was easy to interpret, and the test kit did not require the use of electricity. $6.10,13-16$

The temperature of the children at the time the tests were conducted significantly affected the diagnostic performance of the ICT. In this study, 90.2 percent of 41 children with positive ICT test results were febrile compared with 72.0 percent of 143 who were negative $(p=0.03)$. Studies have shown that large amounts of HRP-II antigens are released duting schizont tupture, which occurs at the peak of a febrile episode. ${ }^{6,10}$ This explanation may account for the higher number of positive ICT results obtained in the febrile children in this study.

For this study, the manufacturers of the ICT test kit donated the test kits used free to the researchers. However, at the time of study, a sole distributor in the state sold each pack of 25 ICT test cards for $\$ 7,000$ (US $\$ 70$ ), with an average cost of about $\$ 280$ (US $\$ 2.8$ ) per test. This unit cost is high when compared to the Giemsa stain microscopy technique with an average cost of $\$ 150$ (US\$1.5) per test (including the cost of microscope slides but excluding the cost of a paid microscopist). In the year 2000 , the ICT was much cheaper at 5,000 (US $\$ 50$ ) per pack of 25 test cards which was thought at that time to be prohibitive by Nigerian standards. ${ }^{12,16}$ However, when compared to the microscopy technique which is time consuming and requires paid expertise, the ICT test cards may be more cost effective. Its simplicity and rapidity would make it a useful tool to be recommended for use at peripheral health centres in the malaria control programme. ${ }^{6}$

This study has demonstrated that the ICT malaria test kit is a rapid diagnostic technique. However, its low sensitivity in children aged $0-5$ years in this study makes it a poor screening tool in the routine diagnosis of falciparum malaria. Nevertheless, it may be useful in establishing a diagnosis of malaria in febrile children above 12 months of age, with features suggestive of severe malaria infection. We recommend that the ICTMálaria $P f \circ$ test be made available at an affordable cost to paediatric emergency units in malaria endemic zones.

\section{Acknowledgements}

We appreciate the contribution of ICT Diagnostics, Sydney, Australia, the manufacturers of the ICT-Malaria Pf ${ }^{\circ}$ kit, who kindly donated the kits for this work. We also acknowledge the support and cooperation of Dr. Awodiji, the medical and nursing staff of the Olikoye Ransome-Kuti Children Emergency Room and the staff of the Paediatric Research Laboratory in LUTH. 


\section{References}

1. UNICEF/WHO. Malaria prevention and treatment. The Prescriber 2000; 18: 1-16.

2. Greenwood B. Malaria mortality and morbidity in Africa. Bull World Health Organ 1999; 77: 617-8.

3. Asagba AO. Editorial. Nig Bull Epidemiol 1991; 1:3.

4. World Health Organisation. A Global Strategy for Malaria Control. Bull World Health Organ 1993; $1: 2$.

5. Chris $\mathrm{CO}$. Rational use of antimalarial drugs. Proceedings of May and Baker Update Symposium. 1992: 15-48.

6. Makler MT, Palmer CJ, Ager AL. A review of practical techniques for the diagnosis of malaria. Ann .Trop Med Parasitol 1998; 92: 419-33.

7. Janssens PG, Wery M. Malaria in Africa south of the Sahara. Ann Trop Med Parasitol 1987; 81: 487-98.

8. Durrheim DN, Govere J, la Grange JJ, Mabuza A. Rapid immunochromatographic diagnosis and Rolling Back Malaria - experiences from an African control program. Afr J Med Med Sci 2001; 30 Suppl: 21-4.

9. Commey JO. Clinical presentation of malaria in Ghana. Ghana Med J 1989; 23: 197- 201.

10. World Health Organisation: $A$ rapid dipstick antigen capture assay for the diagnosis of falciparum malaria. Bull World Health Organ 1996; 74: 47-54.

11. World Health Organisation: Roll Back Malatia. Increasing the momentum. 1999: 1-3.

12. Agomo RU, Akindele SK, Salako LA. Limitations of ParaSight-F as a rapid dipstick antigen capture assay for the detection of Plasmodium faliparum in Nigeria. Nig J Parasitol 1995; 16: 55-64.

13. Cavallo JD, Hernandez E, Gerome P, Plotton N, Debord $T$, Le Vagueresse R. Serum HRP-2 antigens and imported Plasmodium falciparum malaria: comparison of ParaSightF and ICT malaria P.f. Med Trop (Mars) 1997; 57: 353-6.

14. Gaye O, Diouf M, Dansokho EF, McLaughlin G, Diallo S. Diagnosis of Plasmodium falciparum malatia using ParaSight F, ICT malaria PF and malaria IgG CELISA assays. Parasite 1998; 5: 189-92.

15. Thepsamarn P, Prayoollawongsa N, Puksupa P, Puttoom P, Thaidumrong $P$, Wongchai S, Doddara J, Tantayarak ], Buchachart K, Wilairatana P, Looareesuwan S. The ICT Malaria Pf: a simple, rapid dipstick test for the diagnosis of Plasmodium falciparum malaria at the Thai-Myanmar border. Soutb-east Asian J Trop Med Public Healtb 1997; 28: 723-6.

16. Asianya VN, Agomo PU, Okonkwo CA, Mafe $A G$, Akindele
SK, Agomo CO, Aina OO, Okoh HI, Omoruyi SK. Evaluation of a new immunochromatographic test (ICT) for rapid diagnosis of Plasmodium faliparum malatia in Nigeria. Nig J Parasitol 1999; 20: 19-26.

17. Bruce-Chwatt LJ. The malaria parasite. In: Bruce-Chwatt LJ, ed. Essential Malariology. London: William Heinemann Medical Books Ltd, 1985: 77-96.

18. Warhurst D C, Williams J E. Laboratory diagnosis of malaria. J Clin Pathol 1996; 49: 533-8.

19. Beaglehole R, Bonita $R$, Kjellstrom T. Clinical epidemiology. In: Basic Epidemiology. Geneva: World Health Organisation, 1990: 97-105.

20. Olivar M, Develoux M, Chegou Abari AC, Loutan L. Presumptive diagnosis of malaria results in a significant risk of mistreatment of children in urban Sahel. Trans $R$ Soc Trop Med Hyg 1991; 85: 729-30.

21. Trape JF, Peelman P, Morault-Peelman B. Criteria for diagnosing clinical malaria among a semi-immune population exposed to intense and perennial transmission. Trans R Soc Trop Med Hyg 1985; 79: 43542.

22. Stein CM, Gelfand M. The clinical features and laboratory findings in acute Plasmodium falciparum malaria in Harare, Zimbabwe. Cent Afr J Med 1985; 31: 166-70.

23. Redd SC, Kazembe PN, Luby SP, Nwanyanwu O, Hightower AW, Ziba C, Wirima JJ, Chitsulo L, Franco C, Olivar M. Clinical algorithm for treatment of Plasmodium falciparum malaria in children. Lancet 1996; 347: 223-7.

24. Ogala WN. Malaria. In: Azubuike JC, Nkangineme KEO, eds. Paediatrics and Child Health in a Tropical Region. Owerri: African Educational Services, 1999: 426-37.

25. Hendrickse RG. Parasitic Diseases. In: Hendrickse RG, ed. Paediatrics in the Tropics. Oxford: Blackwell Scientific Publications, 1991: 695-710.

26. Shiff CJ, Premji Z, Minjas JN. The rapid manual ParaSight $F$ test. A new diagnostic tool for Plasmodium faliparum infection. Trans R Soc Trop Med Hyg 1993; 87: 646-8.

27. Coleman RE, Maneechai N, Rachapaew N, Kumpitak C, Soyseng V, Miller RS, Thimasarn K, SattabongkotJ. Field evaluation of the ICT Malaria $\mathrm{Pf} / \mathrm{Pv}$ immunochromatographic test for the detection of asymptomatic malaria in a Plasmodium falciparum/vivax endemic area in Thailand. Am J Trop Med Hyg 2002; 66: 379-83. 